\title{
"Giving the gift of life twice": Understanding the lived experiences of parent donors and non-donors in pediatric haploidentical hematopoietic cell transplantation
}

\author{
Megan Schaefer ${ }^{1}$, Vanessa Aguilera ${ }^{1}$, Kendra Parris ${ }^{1}$, Alanna Long ${ }^{2}$, Brandon Triplett ${ }^{3}$, \\ and Sean Phipps ${ }^{3}$ \\ ${ }^{1}$ St Jude Children's Research Hospital \\ ${ }^{2}$ St. Jude \\ ${ }^{3}$ St. Jude Children's Research Hospital
}

September 25, 2021

\begin{abstract}
Background: The use of parental donors in pediatric haploidentical hematopoietic cell transplantation is increasing, but research on the psychosocial impact of parental donation is currently limited. We conducted a retrospective, qualitative study to explore parental perceptions of the donation process and the impact of being a donor (or non-donor) on parents' adjustment and coping with their child's transplant experience. Methods: Parents/caregivers of children who underwent transplantation with a parental donor or a matched unrelated donor $(\mathrm{N}=136)$ participated in interviews and completed an open-ended questionnaire. Both bereaved parents and parents of survivors were surveyed. Results: Six themes were identified in the data: level of understanding and satisfaction; perception of choice; preparation for donation; perceptions of donation and infusion; benefitfinding; and psychological impact of transplantation. Most parents were satisfied with the information they received and reported a good understanding of transplantation and donation procedures. Parents were divided on perspectives of choice, but their responses reflect that the necessity of saving their child's life does not allow for choice. They described considerable effort to prepare for transplantation, physically, emotionally, and logistically. Parents acknowledged the psychological impact while identifying positive outcomes that resulted from their child's transplant journey. Conclusions: Results highlight the unique experiences of parental donors and non-donors from the anticipation phase to the completion of their child's transplant. Additionally, findings inform supportive care guidance by highlighting the need to assess parental donors' emotional functioning, provide support post-donation, and conduct bereavement follow-up.
\end{abstract}

"Giving the gift of life twice": Understanding the lived experiences of parent donors and non-donors in pediatric haploidentical hematopoietic cell transplantation

Megan R. Schaefer ${ }^{1}$, Vanessa Aguilera ${ }^{1}$, Kendra Parris ${ }^{1}$, Alanna Long ${ }^{1}$, Brandon Triplett $^{2}, \&$ Sean Phipps ${ }^{1}$ Department of Psychology.

Department of Bone Marrow Transplantation \& Cellular Therapy St. Jude Children's Research Hospital Memphis, TN, U.S.A.

For Correspondence:

Sean Phipps, Ph.D. Department of Psychology St. Jude Children's Research Hospital 262 Danny Thomas Place Memphis, TN 38105-3678 sean.phipps@stjude.org (901) 595-3603

Word count: 3,246 
Number of tables: 3

Number of references: 27

Conflict of interest: All authors report having nothing to disclose

\title{
Abbreviations
}

HCT Hematopoietic cell transplant

MUD Matched-unrelated donor

HLA human lymphocyte antigen

BMT bone marrow transplant

Haplo haploidentical

G-CSF granulocyte colony stimulating factor

\begin{abstract}
Background: The use of parental donors in pediatric haploidentical hematopoietic cell transplantation is increasing, but research on the psychosocial impact of parental donation is currently limited. We conducted a retrospective, qualitative study to explore parental perceptions of the donation process and the impact of being a donor (or non-donor) on parents' adjustment and coping with their child's transplant experience.
\end{abstract}

Methods: Parents/caregivers of children who underwent transplantation with a parental donor or a matched unrelated donor $(\mathrm{N}=136)$ participated in interviews and completed an open-ended questionnaire. Both bereaved parents and parents of survivors were surveyed.

Results: Six themes were identified in the data: level of understanding and satisfaction; perception of choice; preparation for donation; perceptions of donation and infusion; benefit-finding; and psychological impact of transplantation. Most parents were satisfied with the information they received and reported a good understanding of transplantation and donation procedures. Parents were divided on perspectives of choice, but their responses reflect that the necessity of saving their child's life does not allow for choice. They described considerable effort to prepare for transplantation, physically, emotionally, and logistically. Parents acknowledged the psychological impact while identifying positive outcomes that resulted from their child's transplant journey.

Conclusions: Results highlight the unique experiences of parental donors and non-donors from the anticipation phase to the completion of their child's transplant. Additionally, findings inform supportive care guidance by highlighting the need to assess parental donors' emotional functioning, provide support postdonation, and conduct bereavement follow-up.

Key words: childhood cancer, bone marrow transplantation, donation, parent, bereavement

Despite the growth of donor registries, the majority of children in need of hematopoietic cell transplant (HCT) are without a suitable matched donor ${ }^{1}$. Advances in prevention of Graft versus Host Disease have allowed for a rapid increase in the use of haploidentical (haplo) donor HCTs, which in pediatric patients is typically performed with parent donors ${ }^{2-5}$. For patients without an acceptable matched donor, haploidentical transplantation may be their only chance for a cure. However, such transplants are associated with high rates of morbidity and mortality, especially for patients with relapsed or refractory leukemia and those who have failed a prior transplant 6 .

Most research on the psychosocial impact of HCT donation has focused on unrelated and sibling donors ${ }^{8-12}$. To date, only a single study examining the psychological impact of haploidentical donation for parents has been reported ${ }^{13}$. That qualitative study highlighted four main themes including parental mixed feelings of hope and fear, a need for more information, the desire to do anything for their child, and the impact of transplant outcome on psychosocial functioning ${ }^{13}$. 
Although the literature regarding the effects of parental haploidentical donation is limited, there is relevant research examining the psychological impact of parental solid organ donation to a child. Studies in kidney and liver transplantation with parent donors demonstrate that few experience regret but note an increased risk for anxiety, depression, pain, fatigue, and interpersonal challenges ${ }^{14-18}$. However, psychological benefits to parental donation have also been documented such as less anxiety regarding the procedure compared to non-parental donors and an improved relationship with their child after the transplant ${ }^{19}$. In fact, most parents who were unable to donate reported wishing they could have been the donor to help their child ${ }^{19,20}$.

Research on psychosocial functioning in parental donors/non-donors in the haplo HCT setting is imperative given the increasing frequency of such transplants, along with the vulnerability and potential risk factors associated with serving as a blood/marrow donor. First, parenting a child who undergoes any type of HCT has consistently been described as a stressful and challenging experience ${ }^{21,22}$. Thus, in a situation where a parent also serves as a donor, this stress may be significantly exacerbated due to fulfilling the dual role of donor and caregiver for their ill child. Second, the process of being assessed as a potential donor for one's child who is facing a life-threatening illness may also present unique stressors and psychological challenges. Furthermore, the ultimate choice of a donor may result in both positive and negative consequences for the parent donor as well as the non-donor parent. Finally, because haplo HCTs are more often used in the research setting for the highest risk patients, it may be associated with high rates of morbidity and mortality, parents may face the death of their child, and parents' experiences in the role of donor/non-donor may potentially complicate their bereavement.

Many important questions regarding the psychological impact of parental donation in pediatric HCT remain unaddressed in the literature. Thus, we undertook a retrospective exploration of the experiences of parents/caregivers whose child received a HCT with a parental donor or a matched unrelated donor (MUD). Parents of children who received MUD transplants were also included in the sample as they share many similar experiences, including the anticipation prior to transplant of undergoing HLA typing and awaiting the news of who will be their child's donor. We utilized a qualitative approach, with interviews and a questionnaire with open-ended questions.

\section{Method}

\section{Overview}

These data come from a larger study that included both quantitative and qualitative assessments. The responses to open-ended questions along with the qualitative interviews (questions listed in Table 2) are presented here. As a preparative step for this study, we conducted interviews with seven parents/caregivers (referred to as parents from here on) whose child had received a haplo HCT with a parent donor, survived, and was still receiving follow-up care in our transplant clinic. The objective of the interviews was to inform the development of a transplant donor specific questionnaire that would be administered to a larger number of parents. Subsequently, we surveyed all parents whose child had received a haplo or MUD HCT at St. Jude Children's Research Hospital between January 2006 and September 2017. Both donor and non-donor parents were invited to participate, as were parents of survivors and bereaved parents. Only parents who were English speaking were eligible. Approval from the hospital's Institutional Review Board was obtained prior to recruitment.

\section{Procedure}

The initial qualitative interviews utilized a convenience sample of parents returning for regularly scheduled follow-up visits to the Bone Marrow Transplant clinic. Parents who consented were interviewed by a study team member, with questions designed to explore parental experiences with the process of being evaluated as a possible donor, being informed of the choice of donor, preparation for donation, the actual donation procedures, and subsequent transplant processes. All interviews were audio-recorded and transcribed, and reviewed for content that served to inform the development of a questionnaire that specifically assessed items relevant to parents. Interview questions varied depending on whether the parent was a donor vs. non-donor. However, all questions focused on parental perception of the donation process and the impact of being a 
donor (or non-donor) on their adjustment and coping with their child's transplant experience.

After the development of the donor specific questionnaire, this measure was used as part of an on-line survey. Eligible parents were mailed an invitation explaining the study purpose, rationale, and procedures. Interested parents could access the informed consent and study measures by inputting the website link provided. For parents who did not initially respond, up to three attempts were made to follow-up by telephone. Participants completed a one-time assessment which included the donation-related questionnaire as well as a battery of standardized measures (which will be reported elsewhere). The donation-specific questions were open-ended, with free-text boxes that allowed participants to respond at length.

\section{Analysis}

Qualitative data were analyzed via the framework method, an approach involving identifying a thematic framework, indexing, charting, mapping, and interpretation ${ }^{23}$. All open-ended responses were compiled and collated both by question number and by parental status (e.g., donor status, transplant outcome), creating a matrix whereby all responses could be examined in a case or group by question/theme basis. Five researchers repeatedly reviewed the transcripts and open-ended responses to identify reoccurring concepts in the data. Researchers met to discuss and gain consensus regarding themes of significance. Most of the identified themes emerged directly from the specific demands of the questions, although some emerged spontaneously from the more general questions.

\section{Results}

Of 387 eligible families, 136 parents (26\%) participated, including $37 \%$ parents of survivors and $22 \%$ bereaved parents. Of the non-participants, $37 \%$ were lost to follow-up and were unable to be reached; $32 \%$ were reached but did not respond despite multiple follow-up attempts, and 5\% declined to participate. Our sample included 88 mothers, 44 fathers, and 4 non-parent caregivers. Thirty-two percent of children underwent a haploidentical transplant while $8 \%$ underwent both MUD and haploidentical transplants. Seventy-five percent of participants were non-donors, and $62 \%$ were parents of a child who survived his or her transplant. Additional demographic characteristics for parents and children are reported in Table 1. We identified six major themes in our data. For clarity, results are presented by themes with reference to the questions from whence they were elicited if appropriate (Table 2). Illustrative quotes for each theme are integrated below and in Table 3 .

Theme 1. Level of understanding and satisfaction. Parents were generally satisfied with the information they received regarding their child's transplant and noted feeling as if they had a good level of understanding why the donor choice was made and what to expect during this process. The great majority (95\%) reported feeling well-informed about their child's transplant, regardless of the type of transplant, their role as donor versus non-donor, and the outcome of the transplant. For those few parents voicing dissatisfaction with the provided information, they discussed wishing they had received more anticipatory guidance on the emotional and physical demands of their child undergoing a transplant. Some dissatisfaction was also expressed by parents whose child received a MUD transplant, as they wanted more information about their child's donor and additional treatment options such as the opportunity to donate for their child.

Theme 2. Choice. The response to the yes/no question regarding perception of choice was approximately divided in half ( $54 \%$ yes, $46 \%$ no). However, for those parents who endorsed "yes" as having a choice, their open-ended responses similarly reflected those of parents who said they did not have a choice. As an example, one parent reported having a choice in her child's transplant but contraindicated her original answer when elaborating further, "Yes I did (have a choice) but I could not have chosen different as long as it was the best option for my son". Many parents emphasized having no choice as it was the only option for a chance at saving their child's life, "If death is considered a choice, then I guess there was that option, but it was either treat my child with something or he would die."

Theme 3. Preparation for the donation. Parents reported preparing for the donation in many ways, by researching additional information, making healthier choices, preparing their house (e.g., cleaning to reduce 
the risk of infection), planning the logistics of the admission, spending quality time with loved ones, and praying/maintaining optimism. For donor parents, they discussed focusing on making healthier choices (e.g., nutrition, physical activity) in order to ensure the healthiest version of themselves prior to donation. As one father reported, "I was eating better, making sure that I don't eat any junk and taking super foods. I wanted to be the best that I can so that the stem cells that I might be generating would be the healthiest they could be. Even thinking about and meditating on being healthy and generating good cells." Additionally, donor parents commented on the medical and logistical preparation given the need for mobilization of bone marrow cells with G-CSF. While many parents spoke of the benefits of preparation prior to their child's transplant, some parents described the limitations of preparation, "There is nothing to prepare you for the emotional hell of watching your child go through this process."

Theme 4. Perceptions of donation and infusion. Parents described the opportunity to donate their blood marrow to their child as a great gift. One mother shared, "Not often does a mother get to give her child the gift of life twice. It was a powerful moment to watch $\{$ child\} receive the stem cells. I was filled with hope and love." The process of donation gave parents a sense of control in which they could actively participate to increase their child's chance of survival. As one father reported, "Being able to donate my own stem cells was the first and only opportunity where I could actually do something to help \{patient\} through the process." However, along with the hope and excitement of donating their cells, many parents reported anxiety and fear that their donated cells would fail their child. One mother commented, "What if it doesn't work? What if it gets worse or what if my cells don't take to hers? I guess in a way you feel like it could be your fault, even though it's not. And that's a tough thing to swallow." Furthermore, for those parents who were not matched as a potential donor, some voiced feeling guilt. In regard to MUD transplants, parents expressed immense gratitude to their child's donor for their selfless act of donation.

A few parents highlighted the significance and unexpected anti-climactic nature of the cell infusion. Parents described the cell infusion as a unique experience as they balanced processing the simplicity of the procedure with the meaning and significance of the donated cells. One donor-father expounded, "This is why we are here. They pull out a little five-millimeter syringe. We made jokes and all. Those are damn good-looking stem cells, and everybody laughs. Then one of the nurses just pushes it in. On the one hand, it's the best of what I could muster, but it's also something that could kill her. It couldn't be any more dramatic." Parents of children undergoing MUD transplants explained the intense emotions and limited control felt prior to the infusion as their child's life was now dependent on an unrelated donor.

Theme 5. Benefit-finding. Although not directly asked in the survey, parents reported benefits from their experience. Despite the hardships, many parents identified positive outcomes that resulted from the child's transplant regardless of the disease outcome. These benefits included strengthened parent-child relationships, increased spirituality, greater appreciation for life, new outlooks on life, and the desire to help future families who will undergo this process. Parents voiced appreciation for the time they were able to spend together with their child in the hospital during transplant. A bereaved mother shared, "My relationship with my son was always close but having the opportunity to spend so much time together allowed us the opportunity to talk about deeply personal things. I choose to look at that as a positive side effect of the transplant process."

Theme 6. Psychological impact of transplant. Across the different types of transplant, parents shared feeling mixed emotions throughout the transplant journey, with several using the term 'roller coaster.' One mother elaborated, "This is an awful process to witness, especially when it is your child. There were times that I had to walk out of the room to compose myself or just break down. The physical and emotional toll treatment takes on your child is staggering. There was a spectrum of emotions I felt when I allowed myself to feel them: fear, relief, frustration, sadness, elation, despair, emotional exhaustion, and everything in between." Parents reported other challenges including family separation, marital difficulties, worries about employment, economic strain, adjusting to a new city, and limited time for self-care. They had to continuously make difficult decisions in preparing for the transplant, not only about the medical aspects but logistical issues as well. Parents also often expressed concern about the siblings' coping due to frequent separation from parents and rotating between multiple caregivers. 


\section{Discussion}

Our results highlight the experiences of parental donors and non-donors from the anticipation phase to the completion of their child's HCT, including parents of both children who survived and died from their transplant. Six primary themes emerged from the data including level of understanding and satisfaction, choice, preparation for donation, perceptions of donation and infusion, benefit-finding, and psychological impact of transplant.

Most parents reported feeling satisfied with the information they received prior to transplant. This contradicts previous literature, which indicates parents were uniformly dissatisfied and unprepared for their child's $\mathrm{HCT}^{13}$. Given van Walraven et al.'s (2012) study included participants whose child underwent HCT between 1997-2002, it is possible the level of anticipatory guidance, preparation, and support has significantly expanded since that time. Although our sample was divided in identifying whether parents felt they had a choice in pursuing the transplant, most parents elaborated on the limited nature of their choice. Consistent with prior literature ${ }^{13,19}$, parents who were eligible to donate were unanimous in their decision as they would do anything to save their child's life. This feeling of no choice mirrors research with sibling donors who described their experience as "deliberate no choice," given saying no to donation would result in their sibling's death ${ }^{11}$.

Despite parent donors expressing gratitude for the opportunity to donate, worries regarding potential failure of donor cell engraftment were common. Consistent with prior research, bereaved donors endorsed feelings of guilt, and some perceived the outcome as a personal shortcoming ${ }^{13}$. Similarly, bereaved sibling donors have also voiced guilt, blame, and feeling personally responsible for their sibling's death ${ }^{11}$. On the other hand, some parents who were not chosen to donate also experienced guilt due to being unable to directly help save their child's life. This may be particularly significant given findings from solid organ transplantation suggesting parental non-donors experience greater coping and adjustment challenges than donors ${ }^{19}$, further highlighting the importance of assessing emotional functioning of non-donors throughout the transplant journey and beyond.

Regardless of outcome, parents reported significant impact on family's quality of life due to the intensity of HCT. Common challenges included family separation, marital difficulties, financial strain, and emotional stress. These family stressors are frequently reported in the literature and often impact not only the patients and parents but also the siblings ${ }^{22,24}$, illustrating the importance of family-centered care in this setting. Despite these challenges, many parents, including those who are bereaved, endorsed engaging in benefit-finding during and after their child's transplant. Specifically, strengthened parent-child bonds were identified as a common benefit, which has also been noted among parents of solid organ transplantation ${ }^{19}$. Research with bereaved parents has documented associations between benefit-finding, positive coping, and reductions in complicated grief, suggesting the benefit of providers facilitating discussions focused on meaning-making ${ }^{25-27}$.

A strength of this study involves its novel aims to explore the experiences of both parental donors and nondonors from anticipation to completion of their child's HCT regardless of outcome. However, our findings should be considered in light of several limitations. First, the response rate was low, leaving uncertainty regarding the representativeness of the participating sample. Additionally, this was a single site study, and the sample was relatively homogenous, consisting mainly of participants who identified as white, married, and middle class. There was a broad range of time since transplant with a mean of seven years, introducing the potential of recall bias. Despite these limitations, this is the largest sample studied thus far and provides a fair snapshot of how parents think and process their child's illness experience many years later.

Given this study was cross-sectional and exploratory in nature, longitudinal studies assessing quantitative outcomes such as parental psychosocial functioning, quality of life, and grief with time-points ranging from pre-transplant to long-term follow-up after completion of transplant are needed. Factors impacting recruitment of parental and non-parental stem cell donors should also be explored in order to improve recruitment rates and diverse sampling in future studies. Although our sample primarily included parents of children who survived their HCT, future studies specifically targeting bereaved parent donors/non-donors are warranted. 
The current findings can provide some supportive care guidance for clinicians. Certainly, providing anticipatory guidance prior to the child's transplant appears important to ensuring parents feel satisfied with the information, understand the process, and are prepared for the HCT. Arranging for parents to meet others who have undergone haplo HCT may be beneficial in helping to prepare families for transplant. Specifically, donors may benefit from speaking with other parents who have both donated and fulfilled the caregiving role for the child in the hospital, which is a unique aspect best understood by a parent who has experienced this dual role situation. Interdisciplinary and family-centered psychosocial support should continue throughout and following the child's HCT. As many parents reported, their child's HCT significantly affected the family's quality of life with long-term impacts persisting beyond completion of transplant. This highlights the importance of family-centered care in which the patient's, parents', and siblings' needs are continuously assessed in order to prove appropriate supports for each member's unique psychosocial needs. Specific to this study, there is a great need to assess parental donors' emotional functioning and provide support post-donation, and bereavement follow-up appears particularly important for bereaved donors. As pediatric haplo HCT becomes more common, understanding the experiences of parental donors and non-donors will be critical in ensuring their psychosocial needs are best supported throughout the child's transplant journey.

\section{References}

1. Carreras E, Dufour C, Mohty M, Kröger N. The EBMT handbook: Hematopoietic stem cell transplantation and cellular therapies. Published online 2019.

2. González-Vicent M, Perez MAD. Allogeneic hematopoietic stem-cell transplantation from haploidentical donors using 'ex-vivo'T-cell depletion in pediatric patients with hematological malignancies: State of the art review. Curr Opin Oncol . 2018;30(6):396-401.

3. Ciurea SO, Al Malki MM, Kongtim P, et al. The European Society for Blood and Marrow Transplantation (EBMT) consensus recommendations for donor selection in haploidentical hematopoietic cell transplantation.Bone Marrow Transplant . 2020;55(1):12-24.

4. Aversa F, Pierini A, Ruggeri L, Martelli MF, Velardi A. The evolution of T cell depleted haploidentical transplantation. Front Immunol . 2019;10:2769.

5. Robinson TM, O'Donnell PV, Fuchs EJ, Luznik L. Haploidentical bone marrow and stem cell transplantation: experience with post-transplantation cyclophosphamide. In: Seminars in Hematology . Vol 53. Elsevier; 2016:90-97.

6. Klingebiel T, Cornish J, Labopin M, et al. Results and factors influencing outcome after fully haploidentical hematopoietic stem cell transplantation in children with very high-risk acute lymphoblastic leukemia: Impact of center size: an analysis on behalf of the Acute Leukemia and Pediatric Disease Working Parties of the European Blood and Marrow Transplant group. Blood J Am Soc Hematol . 2010;115(17):3437-3446.

7. Sharma A, Li Y, Huang S, et al. Outcomes of pediatric patients who relapse after first HCT for acute leukemia or MDS. Bone Marrow Transplant . Published online 2021:1-10.

8. Gordon SV, Nivison-Smith I, Szer J, Chapman JR. Volunteer unrelated donor experience after administration of filgrastim and apheresis for the collection of haemopoietic stem cells: the Australian perspective.Intern Med J . 2013;43(11):1183-1190.

9. Burns DS, Robb SL, Phillips-Salimi C, Haase JE. Parental perspectives of an adolescent/young adult stem cell transplant and a music video intervention. Cancer Nurs . 2010;33(4):E20-E27.

10. Switzer GE, Bruce J, Kiefer DM, et al. Health-related quality of life among pediatric hematopoietic stem cell donors. J Pediatr. 2016;178:164-170.

11. MacLeod KD, Whitsett SF, Mash EJ, Pelletier W. Pediatric sibling donors of successful and unsuccessful hematopoietic stem cell transplants (HSCT): A qualitative study of their psychosocial experience. $J$ Pediatr Psychol . 2003;28(4):223-230. 
12. Hutt D, Nehari M, Munitz-Shenkar D, Alkalay Y, Toren A, Bielorai B. Hematopoietic stem cell donation: Psychological perspectives of pediatric sibling donors and their parents. Bone Marrow Transplant . 2015;50(10):1337-1342.

13. Van Walraven SM, Ball LM, Koopman HM, et al. Managing a dual role - experiences and coping strategies of parents donating haploidentical G-CSF mobilized peripheral blood stem cells to their children. Psychooncology . 2012;21(2):168-175.

14. Dew MA, Zuckoff A, DiMartini AF, et al. Prevention of poor psychosocial outcomes in living organ donors: From description to theory-driven intervention development and initial feasibility testing.Prog Transplant . 2012;22(3):280-292.

15. Zeiler K, Guntram L, Lennerling A. Moral tales of parental living kidney donation: A parenthood moral imperative and its relevance for decision making. Med Health Care Philos . 2010;13(3):225-236.

16. Ralph AF, Butow P, Hanson CS, et al. Donor and recipient views on their relationship in living kidney donation: Thematic synthesis of qualitative studies. Am J Kidney Dis . 2017;69(5):602-616.

17. Kruper A, Zanowski SC. Parental live liver donation: Psychosocial considerations in the decision to donate. Curr Opin Organ Transplant . 2015;20(2):140-145.

18. Chen P, Luo Q, Peng L. Anxiety and decreased social support underline poorer quality of life of parent living kidney donors. Asia-Pac Psychiatry . 2015;7(2):197-205.

19. Kärrfelt HM, Berg UB, Lindblad FI, Tydén GE. To be or not to be a living donor: Questionnaire to parents of children who have undergone renal transplantation. Transplantation . 1998;65(7):915-918.

20. Kärrfelt HM, Berg UB, Lindblad FI. Renal transplantation in children: Psychological and donationrelated aspects from the parental perspective. Pediatr Transplant . 2000;4(4):305-312.

21. Lindwall JJ, Russell K, Huang Q, et al. Adjustment in parents of children undergoing stem cell transplantation. Biol Blood Marrow Transplant . 2014;20(4):543-548.

22. Packman W, Weber S, Wallace J, Bugescu N. Psychological effects of hematopoietic SCT on pediatric patients, siblings and parents: A review.Bone Marrow Transplant . 2010;45(7):1134-1146.

23. Gale NK, Heath G, Cameron E, Rashid S, Redwood S. Using the framework method for the analysis of qualitative data in multi-disciplinary health research. BMC Med Res Methodol . 2013;13(1):1-8.

24. Wilkins KL, Woodgate RL. An interruption in family life: Siblings' lived experience as they transition through the pediatric bone marrow transplant trajectory. In: Oncology Nursing Forum . Vol 34. ; 2007.

25. Schaefer MR, Kenney AE, Himelhoch AC, et al. A quest for meaning: A qualitative exploration among children with advanced cancer and their parents. Psychooncology . Published online 2020.

26. Lichtenthal WG, Currier JM, Neimeyer RA, Keesee NJ. Sense and significance: A mixed methods examination of meaning making after the loss of one's child. J Clin Psychol . 2010;66(7):791-812.

27. Wu L, Bonanno G, DuHamel K, et al. Pre-bereavement meaning and post-bereavement distress in mothers of children who underwent haematopoietic stem cell transplantation. Br J Health Psychol . 2008;13(3):419433.

\section{Hosted file}

Hapdon table 1.docx available at https://authorea.com/users/437395/articles/539058--givingthe-gift-of-life-twice-understanding-the-lived-experiences-of-parent-donors-and-nondonors-in-pediatric-haploidentical-hematopoietic-cell-transplantation

\section{Hosted file}


Hapdon table2.docx available at https://authorea.com/users/437395/articles/539058--givingthe-gift-of-life-twice-understanding-the-lived-experiences-of-parent-donors-and-nondonors-in-pediatric-haploidentical-hematopoietic-cell-transplantation

\section{Hosted file}

hapdon table 3.docx available at https://authorea.com/users/437395/articles/539058--givingthe-gift-of-life-twice-understanding-the-lived-experiences-of-parent-donors-and-nondonors-in-pediatric-haploidentical-hematopoietic-cell-transplantation 